\title{
30130 Usefulness of Three-dimensional Technical Stereoscopic Images for Cerebral Endovascular Treatment
}

Ryo Hiramatsu, Shigeru Miyachi, Hiroyuki Ohnishi, Ryokichi Yagi, Shinji Kawabata, and Toshihiko Kuroiwa

Purpose: Reconstructed three-dimensional (3-D) images are essential for cerebral endovascular treatment. Conventional reconstructed 3-D images are no more than planar images projected onto a monitor. In this study, we prepared 3-D stereoscopic images before cerebral endovascular treatment, and conducted preoperative simulation to examine their usefulness (the subjects were six cases with cerebral aneurysms and eight with arteriovenous shunts).

Case Presentations: Case 6: The patient was a 60-year-old male with an unruptured paraclinoid aneurysm. Case 8: The patient was a 64-year-old male with a spinal dural arteriovenous fistula. To prepare 3-D stereoscopic images, 3-D stereoscopic image-converting Work Station/a 3-D monitor was used. The first surgeon performed a preoperative simulation using 3-D stereoscopic images. Subsequently, usual cerebral endovascular treatment was conducted, and the usefulness of 3-D stereoscopic images was evaluated with free comments after surgery.

Conclusion: The application of 3-D stereoscopic images for cerebral endovascular treatment was useful for recognition of spatial orientation. In particular, it was highly assessed in arteriovenous shunt disease patients with a complex vascular structure. Therefore, 3-D stereoscopic images may be appropriate for training for non-skilled specialists in cerebral endovascular treatment and student education.

Keywords \ 3-D rotational angiography, cerebral aneurysm, cerebral arteriovenous malformation, dural arteriovenous fistula

\section{Introduction}

Recently, three-dimensional (3-D) stereoscopic images have commonly been used for surgery. In particular, many studies reported their usefulness in the field of laparoscopic surgery. ${ }^{1-3)}$ Recently, 3-D stereoscopic images have also been used for a surgery-assisting robot, "da Vinci," and adopted in many institutions in Japan. ${ }^{4-6)}$ Furthermore, 3-D stereoscopic images in combination with a surgery-assisting navigation system for neuroendoscopic surgery ${ }^{7}$ or microscopic surgery were also introduced in the field of neurosurgery, and several studies indicated their usefulness. ${ }^{8-11)}$

For cerebral endovascular treatment, 3-D rotational angiography (3-D RA) is essential as a diagnostic tool.

Department of Neurosurgery and Endovascular Therapy, Osaka Medical College, Takatsuki, Osaka, Japan

Received: March 23, 2016; Accepted: July 29, 2016

Corresponding author: Ryo Hiramatsu. Department of Neurosurgery and Endovascular Therapy, Osaka Medical College, 2-7, 072683-1221, 2-7 Daigaku-machi, Takatsuki, Osaka 569-8686, Japan

Email:neu106@osaka-med.ac.jp

(C2016 The Editorial Committee of Journal of Neuroendovascular Therapy. All rights reserved.
However, 3-D reconstructed images made from 3-D RA (3-D images) are no more than planar images. In this study, we applied 3-D stereoscopic images for cerebral endovascular treatment, and examined their usefulness based on surgeons' evaluation.

\section{Case Presentation}

\section{Materials and methods}

The subjects consisted of 6 cases with cerebral aneurysms (unruptured: 5 cases, ruptured: 1 case) and 8 with arteriovenous shunts with a complex vascular structure (cerebral arteriovenous malformation (AVM): 2 cases, dural arteriovenous fistula (dAVF): 3 cases, and spinal AVF: 3 cases) (total: 14 cases) (Table 1). 3-D stereoscopic images were prepared using a surgery-assisting imaging system (Plissimo XV) developed by Panasonic Medical Solutions Co., Ltd. Concerning procedures to prepare 3-D stereoscopic images, the raw data obtained on 3-D RA before cerebral endovascular treatment are initially output to a CD-R in the DICOM (Digital Imaging and Communication in Medicine) mode, and open on the Plissimo XV system. 3-D stereoscopic images are completed in a few 


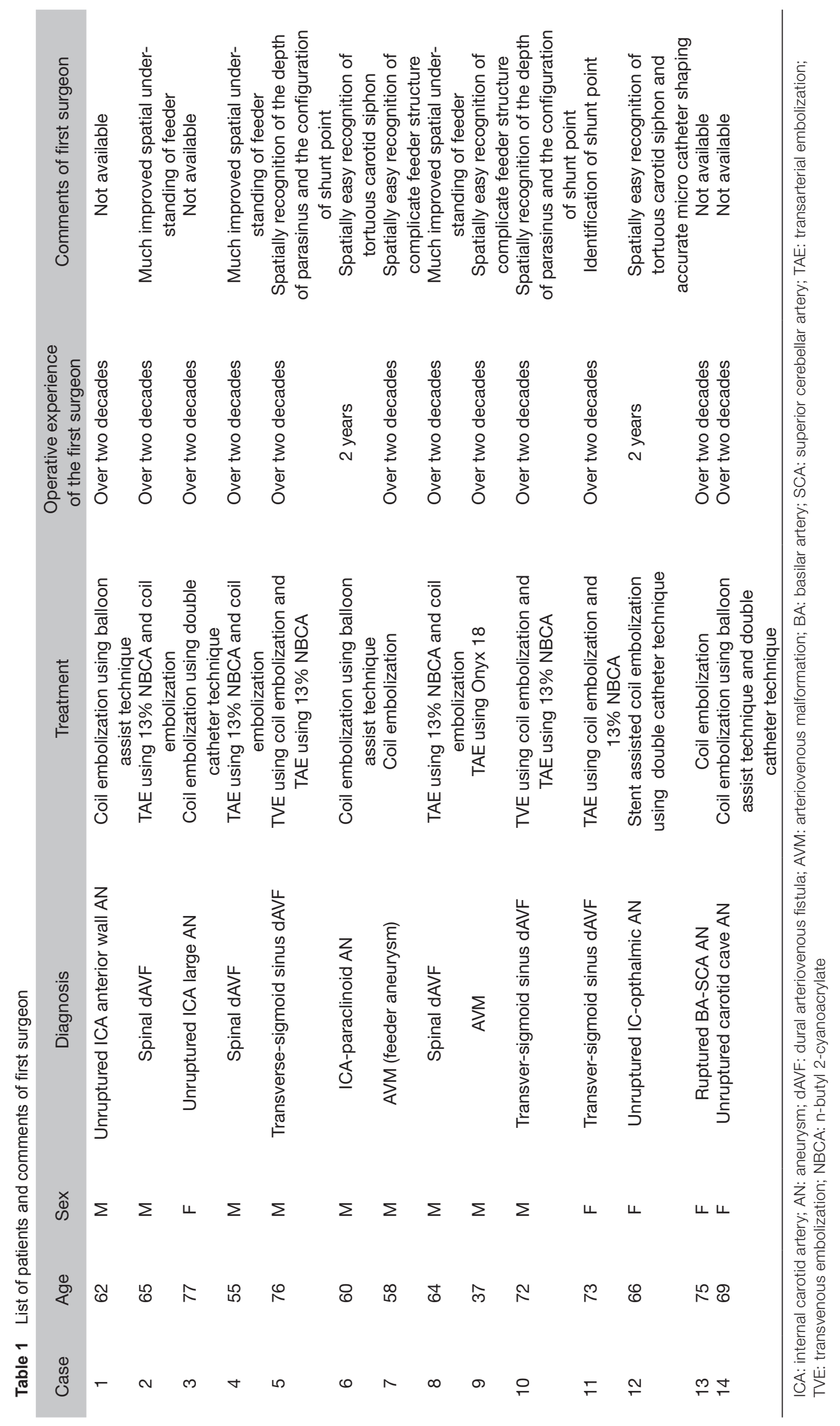




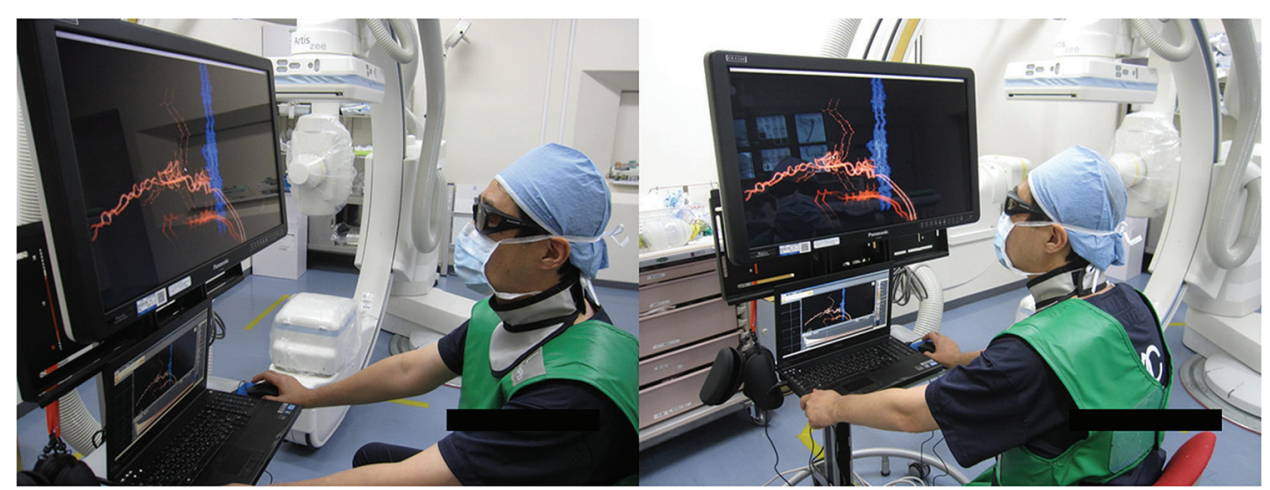

Fig. 1 First surgeon runs a preoperative simulation on access to lesion and depth of lesion etc. using 3-D stereoscopic system.

seconds, and projected onto a 3-D stereoscopic imagematched liquid crystal display (LCD) monitor developed by Panasonic Medical Solutions Co., Ltd. The surgeon wore polarized glasses for 3-D images, and conducted preoperative simulation of an access to the lesion or the lesion depth using stereoscopic images while moving a mouse on the monitor (Fig. 1). Subsequently, usual treatment was started. After treatment, the first surgeon evaluated the therapeutic usefulness of preoperative simulation using 3-D stereoscopic images with free comments.

\section{Results}

In all 14 cases, 3-D stereoscopic images could be readily prepared using the Plissimo XV system. With respect to the usefulness of 3-D stereoscopic images for cerebral endovascular treatment, the surgeon indicated that these images were useful for recognition of spatial orientation involving the vascular structure in the deep direction and branches duplicated with working angle. Concretely, it was possible to get the picture as the torsion of a flexed vessel and the distance between two adjacent blood vessels, which may be missed on a planar image in the presence of AVM. In the presence of a dAVF at the transverse sinus, the depth of several parasinuses and shape of the junction were threedimensionally visualized, and 3-D stereoscopic images were useful for determining an approaching angle and direction. In cerebral aneurysms, the usefulness of 3-D stereoscopic images in comparison with standard treatment was not demonstrated when the surgeon was a skilled instructor regarding cerebral endovascular treatment. This was possibly because the vascular structure of cerebral aneurysms was not complex, differing from that of shunt disease, facilitating 3-D structure imaging; the usefulness for skilled instructors was not demonstrated. However, when the surgeon was a non-skilled specialist in cerebral endovascular treatment, the depth of an access route to the cerebral aneurysm (tortuous shape at the siphon region) and shaping of a microcatheter (MC) could be more accurately clarified. However, there was a time lag between manual operations on a mouse and stereoscopic image motions on a 3-D stereoscopic image-matched LCD monitor; some surgeons felt stressed.

\section{Case 6}

Patient: A 60-year-old male.

Present illness: He consulted a local clinic with dizziness. Head magnetic resonance imaging (MRI)/magnetic resonance angiography (MRA) revealed a left internal carotid artery aneurysm. He was referred to our hospital. In our department, cerebral angiography was performed. The maximum diameter of the aneurysm exceeded $5 \mathrm{~mm}$. Based on the age/size and his wishes, coil embolization was selected. Treatment: The location was paraclinoid aneurysm (Fig. 2A), with a maximum diameter of $5.30 \mathrm{~mm}$. The neck diameter was $3.95 \mathrm{~mm}$, being relatively broad (dome/neck ratio: 1.35). Balloon-assisted coil embolization was selected for the following reasons: paraclinoid aneurysm, the distance from the siphon region was very short, making it difficult to insert a $\mathrm{MC}$ into the aneurysm (engagement); and an $\mathrm{MC}$ was expected to be unstable during coil embolization. Treatment was performed under general anesthesia. A 6 Fr guiding catheter (GC) was inserted into the left cervical internal carotid artery (C1 level). Subsequently, the raw data obtained on preoperative 3-D RA were output to a CD-R in the DICOM mode, and opened on the Plissimo XV system. 3-D stereoscopic images were completed in approximately 10s (Fig. 2B). After the surgeon (nonskilled specialist in cerebral endovascular treatment) freely operated them on a 3-D stereoscopic image-matched LCD monitor using a mouse for preoperative simulation, he conducted $\mathrm{MC}$ shaping and engagement. A total of five 


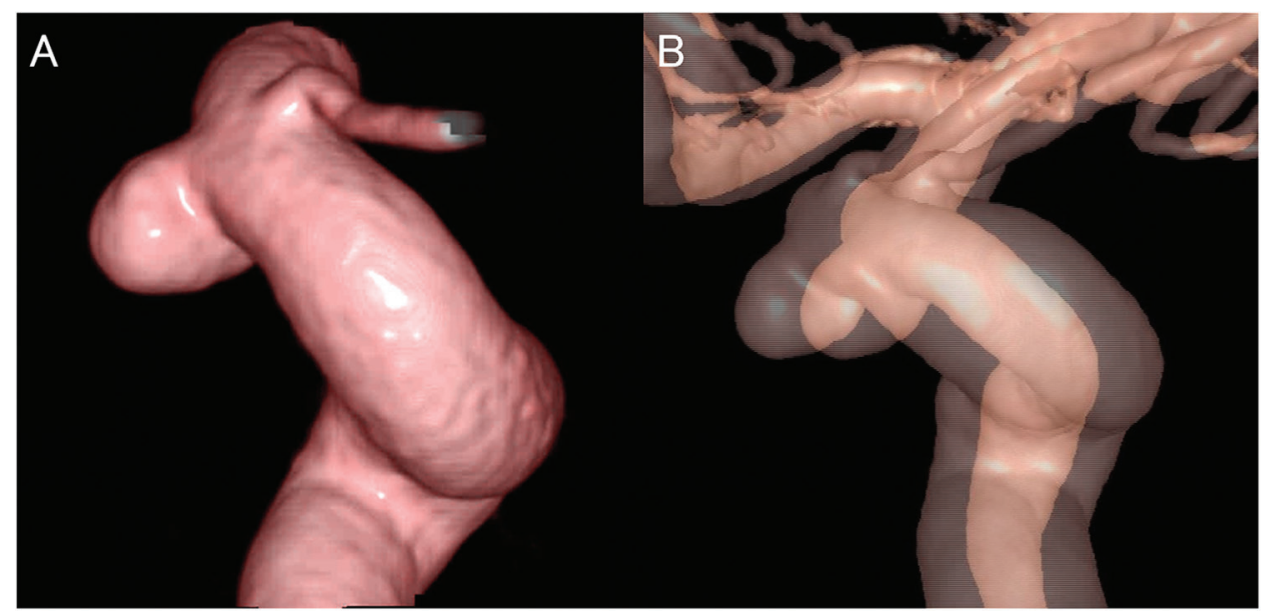

Fig. 2 Case 6. (A) Pre-operative 3-D rotational angiography (left internal carotid angiogram, antero-posterior view). (B) Pre-operative 3-D stereoscopic angiography (left internal carotid angiogram, anteroposterior view).

coils were used, and final angiography confirmed complete obliteration of the aneurysm.

Evaluation: The depth of the siphon region was considered to be deeper than recognized on standard 3-D RA. For this reason, a MC three-dimensionally preshaped in accordance with 3-D RA findings was guided by converting the direction with a microguidewire. At this point, a MC was guided while visually evaluating or feeling wire resistance at the siphon region, but the depth of the siphon region was confirmed before surgery; therefore, engagement could be achieved without excessive wire operations while more sensitively feeling their resistance.

\section{Case 8}

Patient: A 64-year-old male.

Present illness: As weakness of the bilateral lower limbs and bladder and rectal disturbance had persisted for 4 months, he was admitted to the Department of Orthopedics in our hospital to undergo surgery for hernia of the intervertebral disk. Preoperative MRI of the lumbar vertebrae revealed a flow void and dilated abnormal blood vessel at the thoracolumbar vertebral level; therefore, he was referred to our department. Spinal angiography showed a spinal dAVF feeding by the intercostal artery at the seventh to eighth right thoracic vertebral level (Fig. 3A). Endovascular treatment was selected.

Treatment: As described for Case six, the raw data obtained on spinal angiography (3D-RA) before treatment were output to a CD-R in the DICOM mode, and developed on the Plissimo XV system. 3-D stereoscopic images were completed in approximately 10s (Fig. 3B). This patient had a spinal dAVF. The drainer and feeder (T7, T8 radiculomedullary artery) were overlapped with individual stereoscopic images, respectively. After the surgeon (skilled instructor regarding cerebral endovascular treatment) conducted preoperative simulation on a 3-D stereoscopic image-matched LCD monitor, he started treatment. Under general anesthesia, a GC was inserted into the right $\mathrm{T} 7$ intercostal artery using $6 \mathrm{Fr}$ and $4 \mathrm{Fr}$ coaxial systems. Subsequently, the surgeon attempted to insert a MC into the radiculomedullary artery as the feeder, but preoperative 3-D stereoscopic images showed a sharp branching angle from the intercostal artery; therefore, the post-vascular-branching intercostal artery (not feeder) was occluded by coil without excessive wire operations. Subsequently, n-butyl 2-cyanoacrylate (NBCA) was infused before the vascular branch, and the infusion of NBCA into only the feeder was possible. NBCA was also infused through the right T8 radiculomedullary artery. Final angiography confirmed the complete disappearance of the spinal dAVF.

Evaluation: The branching angle of the T7 radiculomedullary artery was sharp, which had been confirmed on 3-D images. However, the angle of the vascular branch on the feeder's side was sharp, with a horizontal hairpin turn; it was possible to accurately evaluate such 3-D information (Fig. 3). This facilitated the establishment of an appropriate therapeutic strategy without excessive wire operations.

\section{Discussion}

The history of stereoscopic images is long, and stereoscopic imaging with two films has commonly been conducted 


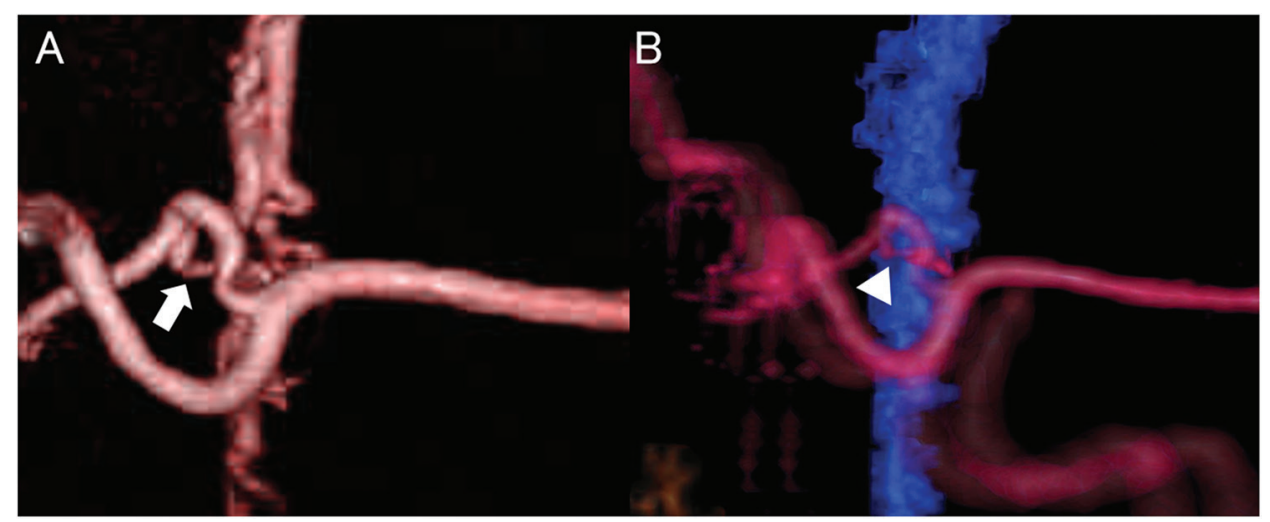

Fig. 3 Case 8. (A) Pre-operative 3-D rotational angiography (right 7th intercostal angiogram, anteroposterior view). Arrow shows a hairpin curve of feeder on 3-D rotational angiography. (B) Pre-operative 3-D stereoscopic angiography (right 7th intercostal angiogram, antero-posterior view). Arrow head shows a hairpin curve of feeder on 3-D stereoscopic angiography.

since the beginning of the 20th century, when simple angiography became possible. ${ }^{12-14)}$ Furthermore, Saito et al. reported three-dimensional single-shot angiography in 1930. This procedure made it possible to obtain 3-D images through two sessions of single-shot imaging at different $\mathrm{X}$-ray-tube angles, but required two sessions of contrast-medium infusion. To facilitate 3-D shooting on one session of serial angiography, it was necessary to transfer a single X-ray tube between two positions several times in a short period, ${ }^{15-17)}$ alternately use two X-ray tubes, ${ }^{18,19)}$ or adopt a single $\mathrm{X}$-ray tube with 2 focal points (dual-focus $\mathrm{X}$-ray tube). ${ }^{20)}$ As there were limitations, such as resolution, a method to perform the same type of serial angiography twice by slightly changing a specific angle (convergent angle: 6 degrees to 12 degrees) using a single X-ray tube with one focal point (single-focus X-ray tube) was again commonly used. However, the limitation of two sessions of contrast-medium infusion was not resolved. As a routine method, 2-directional images at different angles were displayed, and a gaze point was regulated in proximal or distal directions using the crossing or parallel methods for unaided vision-based stereoscopic vision.

The 3-D stereoscopic imaging system used in this study facilitates the preparation of stereoscopic images based on raw data on 3-D RA prior to cerebral endovascular treatment. Anyone wearing 3-D polarized glasses can confirm stereoscopic images. Therefore, it is not necessary to perform two sessions of angiography for preparing 3-D stereoscopic images, and skills to make an interpretation of stereoscopic images are also unnecessary. In this study, 3-D stereoscopic images could be readily prepared in a few seconds in all patients. In addition, these images were useful for evaluating the vascular structure and selecting therapeutic strategies before surgery. In particular, they were useful for evaluating the vascular anatomy and feederdrainer positional relationship/distance in the shunt disease; their application may prevent complications related to vascular-course underestimation-associated excessive operations and a prolonged treatment-related increase in the exposure dose. On the other hand, there was a time lag between manual operations on a mouse and stereoscopic image motions on a 3-D stereoscopic image-matched LCD monitor, raising an issue regarding operability. In the future, software and hardware should be further improved.

\section{Conclusion}

In this study, we used 3-D stereoscopic images for the preoperative simulation of cerebral endovascular treatment, and examined their usefulness. It was easy to prepare 3-D stereoscopic images using 3-D stereoscopic imageconverting Work Station/a 3-D monitor. Furthermore, 3-D stereoscopic images facilitated the assessment of spatial orientation in the presence of arteriovenous shunts with a complex vascular structure, suggesting their usefulness. Spatial orientation is an important factor for cerebral endovascular treatment, and the preoperative confirmation of 3-D stereoscopic images may provide information useful for training for non-skilled physicians specializing in cerebral endovascular treatment or student education.

No study has introduced 3-D stereoscopic images for the preoperative simulation of cerebral endovascular treatment, which we performed. We submitted an application to the Ethics Review Board of our hospital. In the future, a prospective study should be conducted to demonstrate the usefulness of 3-D stereoscopic images. 


\section{Disclosure Statement}

There is no conflict of interest to be disclosed regarding this article.

\section{References}

1) Kong $\mathrm{SH}$, Oh BM, Yoon H, et al: Comparison of two- and three-dimensional camera systems in laparoscopic performance: a novel 3D system with one camera. Surg Endosc 2010; 24: 1132-1143.

2) Silvestri M, Simi M, Cavallotti C, et al: Autostereoscopic three-dimensional viewer evaluation through comparison with conventional interfaces in laparoscopic surgery. Surg Innov 2011; 18: 223-230.

3) LaGrange CA, Clark CJ, Gerber EW, et al: Evaluation of three laparoscopic modalities: robotics versus threedimensional vision laparoscopy versus standard laparoscopy. J Endourol 2008; 22: 511-516.

4) Ito M, Asano $Y$, Shimizu T, et al: Comparison of standard laparoscopic distal pancreatectomy with minimally invasive distal pancreatectomy using the da Vinci S system. Hepatogastroenterology 2014; 61: 493-496.

5) Yoshida S, Fukui N, Saito K, et al: Novel image monitoring system using a head-mounted display for assistants in da Vinci surgery. Int J Urol 2015; 22: 520-521.

6) Fujiwara K, Fukuhara T, Niimi K, et al: Load evaluation of the da Vinci surgical system for transoral robotic surgery. J Robot Surg 2015; 9: 315-319.

7) Inoue $\mathrm{D}$, Yoshimoto $\mathrm{K}$, Uemura $\mathrm{M}$, et al: Three-dimensional high-definition neuroendoscopic surgery: a controlled comparative laboratory study with two-dimensional endoscopy and clinical application. J Neurol Surg A Cent Eur Neurosurg 2013; 74: 357-365.

8) Rohde V, Hans FJ, Mayfrank L, et al: How useful is the 3-dimensional, surgeon's perspective-adjusted visualisation of the vessel anatomy during aneurysm surgery? A prospective clinical trial. Neurosurg Rev 2007; 30: 209-217.

9) Unsgaard G, Ommedal S, Rygh OM, et al: Operation of arteriovenous malformations assisted by stereoscopic navigationcontrolled display of preoperative magnetic resonance angiography and intraoperative ultrasound angiography. Neurosurgery 2007; 61: 407-415; discussion 415-416.

10) Mathiesen T, Peredo I, Edner G, et al: Neuronavigation for arteriovenous malformation surgery by intraoperative three-dimensional ultrasound angiography. Neurosurgery 2007; 60: 345-350; discussion 350-351.

11) Kockro RA, Reisch R, Serra L, et al: Image-guided neurosurgery with 3-dimensional multimodal imaging data on a stereoscopic monitor. Neurosurgery 2013; 72: 78-88.

12) Ohta $T$, Nagata $S$, Deguchi J, et al: Three-dimensional image atlas of the cranium/brain/blood vessels. Kinpodo. Kyoto, 1995.

13) Washburn MF: Retinal rivalry as a neglected factor in stereoscopic vision. Proc Natl Acad Sci USA 1933; 19: 773-777.

14) Fray WW, Warren SL: Stereoscopic Röntgenography of the Breasts: An Aid in Establishing the Diagnosis of Mastitis and Carcinoma. Ann Surg 1932; 95: 425-432.

15) Takahashi M, Tamakawa Y, Goto K, et al: Serial cerebral angiography in stereoscopic magnification. AJR Am J Roentgenol 1976; 126: 1211-1218.

16) Vogelsang H, Dietz K: Stereoscopic magnification in spinal angiography. AJNR Am J Neuroradiol 1983; 4: 588-589.

17) Mokrohisky JF, Murtagh F, Paul RE, et al: Biplane stereoscopic cerebral angiography. Acta Radiol 1956; 46: 262-272.

18) Fernstrom I, Lindblom K: Simultaneous stereoangiography. Acta Radiol 1955; 44: 230-232.

19) Tobe $T$, Saito $S$, Toda $N$, et al: Simultaneous stereoroentogenography. Gunma J Med Sci 1966; 15: 23-31.

20) Doi K, Rossmann K, Duda EE: Application of longitudinal magnification effect to magnification stereoscopic angiography: a new method of cerebral angiography. Radiology 1977; 124: 395-401. 